\title{
Engineering Principles of Synthetic Biochemical Oscillators with Negative Cyclic Feedback
}

\author{
Yutaka Hori and Richard M. Murray
}

\begin{abstract}
In this paper, we analyze the oscillatory dynamics of a class of cyclic gene regulatory networks and provide engineering principles for the robust synthesis of biochemical oscillators. We first review the first author's previous result that the oscillatory parameter regime of the gene regulatory circuits can be rigorously explored by the local stability analysis of a unique equilibrium. The local stability analysis then leads to the first engineering principle that the circuit components, or genes, should be chosen so that the kinetic profiles of the circuit components are similar to each other. Using a homogeneous oscillator model, we further discuss how to reduce the cellto-cell variability of the oscillators that is caused by intrinsic noise.
\end{abstract}

\section{INTRODUCTION}

Construction of synthetic biochemical oscillators requires deep understanding of both nonlinearity and feedback in biochemical process. The reliable engineering of oscillators is an important milestone towards robust synthesis of more complex dynamical circuits in synthetic biology. Starting with Repressilator [1], a number of synthetic biochemical oscillators were implemented in the last 15 years with the help of mathematical model and intuition (see [2]-[4], for example).

The first dynamical model of a genetic oscillator was presented by Goodwin [5], where a negative autoregulation of a single gene was modeled and analyzed, in 1965, shortly after the discovery of lac operon [6]. This model was named as Goodwin oscillator, and many versions of the Goodwin oscillator were proposed and analyzed in later years using local stability analysis and harmonic balancing technique (see [7]-[11], for example). More recently, general analysis frameworks were developed to study oscillations as well as stability of a class of gene regulatory networks with multiple genes, using the monotone property [12] and passivity [13], [14] of the dynamics.

In another line of research, the first author and his colleagues [15], [16] proposed a multi-agent modeling framework that allows us to analytically characterize the oscillation parameter regime of cyclic gene regulatory networks including Repressilator [1]. The analytic characterization was useful to gain biological insights on how the combination of genetic parts such as promoter, ribosome binding sites (RBS) and coding sequence (CDS) affect the dynamics.

This work was supported in part by the Defense Advanced Research Projects Agency (DARPA) under Living Foundries program (HR0011-12C-0065). Y. Hori is supported by JSPS Fellowship for Research Abroad.

Y. Hori and R. M. Murray are with Department of Computing and Mathematical Sciences, California Institute of Technology, USA. yhoriecaltech.edu, murrayecds.caltech.edu

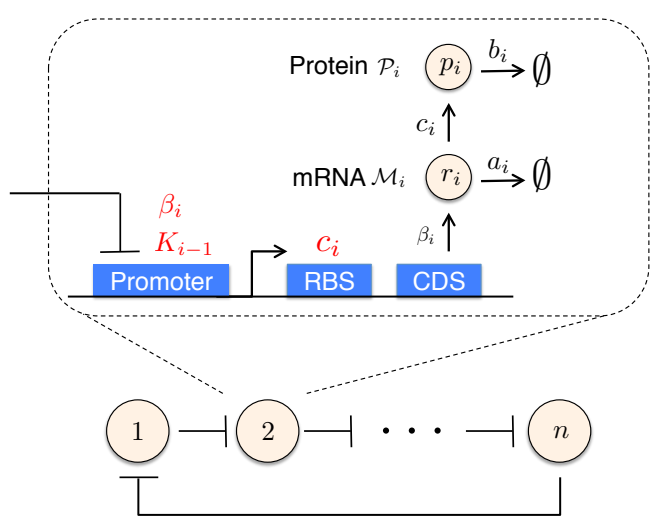

Fig. 1. Schematic diagram of cyclic gene regulatory network

The previous work, however, had limitation from a practical point of view due to technical assumptions such as that the dynamics of gene expression are homogeneous for all genes (homogeneous assumption).

Motivated by this situation, this paper extends the previous analytic work [15] and provides more practical insight for the design of biochemical oscillators with negative cyclic feedback. Specifically, we first explore the oscillation parameter regime without the homogeneous assumption and show that it is easier to produce oscillations when the dynamics of expression are balanced, or homogeneous, between genes. Then, using the homogeneous assumption, we analyze the dependence of the equilibrium point on reaction parameters. This analysis provides circuit design strategies to reduce intrinsic noise, which comes from low-copy-number nature of molecules in a cell. Moreover, we illustrate that the design space of promoters and RBSs can be systematically determined using a lower dimensional parameter space of summarized parameters.

The organization of this paper is as follows. In Section II, we first introduce a mathematical model of cyclic gene regulatory networks. Then, Section III analyzes the oscillation parameter regime without the homogeneous assumption. In Section IV, we analyze how the combinations of promoters and RBSs affect the level of intrinsic noise. Finally, Section $\mathrm{V}$ summarizes our findings and concludes the paper.

\section{Mathematical Model of Biochemical OSCILLATORS}

We consider gene regulatory networks where $n$ different genes, $\mathcal{G}_{1}, \mathcal{G}_{2}, \cdots, \mathcal{G}_{n}$ interact in a cyclic way as illustrated in Fig. 1. As the figure shows, a gene $\mathcal{G}_{i}$ transcribes the 
TABLE I

STOICHIOMETRY AND PROPENSITY FUNCTIONS

\begin{tabular}{|c|c|c|}
\hline Reaction & Stoichiometry & Propensity \\
\hline Transcription of $\mathcal{M}_{i}$ & $\boldsymbol{s}_{1}=[1,0]^{T}$ & $\beta_{i} \frac{K_{i-1}^{\nu_{i}}}{K_{i-1}^{\nu_{i}}+p_{i-1}^{\nu_{i}}}$ \\
Translation of $\mathcal{M}_{i}$ & $\boldsymbol{s}_{2}=[0,1]^{T}$ & $c_{i} r_{i}$ \\
Degradation of $\mathcal{M}_{i}$ & $\boldsymbol{s}_{3}=[-1,0]^{T}$ & $a_{i} r_{i}$ \\
Degradation of $\mathcal{P}_{i}$ & $\boldsymbol{s}_{4}=[0,-1]^{T}$ & $b_{i} p_{i}$ \\
\hline
\end{tabular}

messenger RNA (mRNA) molecule $\mathcal{M}_{i}$ and the mRNA molecule is translated into the protein molecule $\mathcal{P}_{i}(i=$ $, 1,2, \cdots, n)$. The protein molecule $\mathcal{P}_{i}$ then represses the transcription the subsequent mRNA $\mathcal{M}_{i+1}$ by blocking the promoter region of the gene $\mathcal{G}_{i+1}$ for $i=1,2, \cdots, n-1$, and the last protein in the chain $\mathcal{P}_{n}$ represses $\mathcal{G}_{1}$ to form a cyclic feedback (see Fig. 1). This class of gene regulatory network is called cyclic gene regulatory network [15] and Repressilator [1] is an example of experimentally synthesized cyclic gene regulatory network with $n=3$ genes.

Let $\boldsymbol{x}_{i}:=\left[r_{i}, p_{i}\right]^{T} \in \mathbb{R}_{+}^{2}$ denote the concentrations of mRNA and protein molecules produced from gene $\mathcal{G}_{i}(i=$ $1,2, \cdots, n)$. The stoichiometry and the propensity function of transcription, translation and degradation of the $i$ th mRNA $\mathcal{M}_{i}$ and protein $\mathcal{P}_{i}$ are summarized in Table I, where $a_{i}$ and $b_{i}$ represent the degradation rates of $\mathcal{M}_{i}$ and $\mathcal{P}_{i}$ including the dilution due to cell division, respectively, and $c_{i}$ and $\beta_{i}$ are the translation and transcription rates, respectively. The constant $K_{i-1}$ and $\nu_{i}$ are the MichaelisMenten constant and the Hill coefficient associated with the protein $\mathcal{P}_{i-1}$ and the corresponding promoter on gene $\mathcal{G}_{i}$. Using these definitions, the dynamics of the mRNA and protein concentrations, $r_{i}$ and $p_{i}$, can be written as

$$
\dot{\boldsymbol{x}}_{i}=S \boldsymbol{w}_{i}\left(\boldsymbol{x}_{i}, p_{i-1}\right) \quad(i=1,2, \cdots, n)
$$

where the stoichiometry matrix $S$ and the propensity function $\boldsymbol{w}_{i}\left(\boldsymbol{x}_{i}, p_{i-1}\right)$ are defined by

$$
\begin{aligned}
& S:=\left[s_{1}, s_{2}, s_{3}, s_{4}\right]=\left[\begin{array}{cccc}
1 & 0 & -1 & 0 \\
0 & 1 & 0 & -1
\end{array}\right], \\
& \boldsymbol{w}_{i}:=\left[\begin{array}{llll}
\beta_{i} \frac{K_{i-1}^{\nu_{i}}}{K_{i-1}^{\nu i}+p_{i-1}^{\nu i}} & c_{i} r_{i} & a_{i} r_{i} & b_{i} p_{i}
\end{array}\right]^{T} \text {. }
\end{aligned}
$$

In order to avoid notational clutter, we use subscripts 0 and $n+1$ as substitution of $n$ and 1 , respectively, throughout the paper. Note that the propensity function $\boldsymbol{w}_{i}$ depends on $p_{i-1}$, which is the concentration of a protein produced by the precedent gene $\mathcal{G}_{i-1}$.

The entire cyclic gene regulatory network can then be modeled by

$$
\dot{\boldsymbol{x}}=\left(I_{n} \otimes S\right) \boldsymbol{w}
$$

with the molecular concentrations $\boldsymbol{x}:=$ $\left[\boldsymbol{x}_{1}^{T}, \boldsymbol{x}_{2}^{T}, \cdots, \boldsymbol{x}_{n}^{T}\right]^{T}=\left[r_{1}, p_{1}, r_{2}, p_{2}, \cdots, r_{n}, p_{n}\right] \in \mathbb{R}_{+}^{2 n}$ and the propensity function $\boldsymbol{w}:=\left[\boldsymbol{w}_{1}^{T}, \boldsymbol{w}_{2}^{T}, \cdots, \boldsymbol{w}_{n}^{T}\right]^{T}$. The symbol $\otimes$ stands for the Kronecker product of matrices.
The equation (4) can be more specifically written as

$$
\begin{aligned}
& \dot{r}_{i}=-a_{i} r_{i}+\beta_{i} \frac{K_{i-1}^{\nu_{i}}}{K_{i-1}^{\nu_{i}}+p_{i-1}^{\nu_{i}}}, \\
& \dot{p}_{i}=-b_{i} p_{i}+c_{i} r_{i}
\end{aligned}
$$

for $i=1,2, \cdots, n$.

In synthetic oscillator design, one of the goals of a circuit designer is to find a combination of genetic parts such as promoters, ribosome binding sites (RBS) and coding sequences (CDS) from a parts library so that the parameters of the circuit fall into oscillation regime. As illustrated in Fig. 1, the choice of the promoter region mainly affects transcription rates $\beta_{i}$, Michaelis-Menten constant $K_{i}$ and Hill coefficient $\nu_{i}$, and the choice of RBS affects the translation rate $c_{i}$. A well-characterized family of these genetic parts has been recently available for the purpose of biocircuit design [17], [18]. The degradation and dilution rates $a_{i}$ and $b_{i}$ largely depends on cell division dynamics, and we here assume that $a_{i}$ and $b_{i}$ are not tunable.

In what follows, we analyze the cyclic gene regulatory network system (5) and provide guidelines on how one should choose promoters and RBS to make robust oscillators, along with theoretical insights. More specifically, in the next section, we explore the question that whether the parameters of each genetic part, $c_{i}, \beta_{i}$ and $K_{i}$, should be similar or different between genes or not to produce oscillations.

\section{Analysis of Gene-to-Gene Heterogeneity}

\section{A. Existence of oscillations}

It is known that the dynamics of the cyclic gene regulatory network has oscillations only if there are an odd number of genes, $n$, in the network [19]. In other words, the loop gain of the system needs to be negative for oscillations. Thus, we assume that $n$ is odd throughout the paper.

Assumption 1. We assume the number of genes in the network, $n$, is odd.

In Hori et al. [15], it was shown that the existence of oscillations in cyclic gene regulatory networks can be rigorously verified by local stability analysis around a unique equilibrium of the system based on the idea of PoincaréBendixson type theorem for cyclic systems [20].

Proposition 1. [15] The gene regulatory network (5) has a unique equilibrium point. Moreover, the system has periodic oscillations if and only if the unique equilibrium point is locally unstable.

This proposition recasts the question that how the effect of gene-to-gene heterogeneity affects the existence of oscillations into the study of instability parameter regime of an equilibrium point. For stability analysis, we linearize the system (5) around a unique equilibrium point. Then, we have the following lemma showing the characteristic polynomial of the linearized system.

Lemma 1. Let $\boldsymbol{x}^{*}:=\left[r_{1}^{*}, p_{1}^{*}, r_{2}^{*}, p_{2}^{*}, \cdots, r_{n}^{*}, p_{n}^{*}\right] \in \mathbb{R}_{+}^{2 n}$ denote the unique equilibrium point of the cyclic gene 


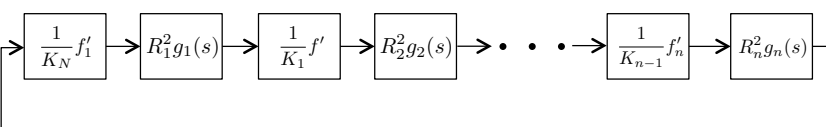

Fig. 2. Block diagram of the linearized cyclic gene regulatory network system

regulatory network (5). The characteristic polynomial of the linearized system is given by

$$
\prod_{i=1}^{n}\left(T_{a_{i}} s+1\right)\left(T_{b_{i}} s+1\right)-\prod_{i=1}^{n} \frac{R_{i}^{2}}{K_{i}} f_{i}^{\prime}\left(\frac{p_{i-1}^{*}}{K_{i-1}}\right)=0,
$$

where

$$
T_{a_{i}}:=\frac{1}{a_{i}}, T_{b_{i}}:=\frac{1}{b_{i}}, R_{i}^{2}:=\frac{c_{i} \beta_{i}}{a_{i} b_{i}}, f_{i}(p):=\frac{1}{1+p^{\nu_{i}}},
$$

and $f^{\prime}(\cdot)$ stands for the derivative of $f(\cdot)$ with $p$. The equilibrium point is locally unstable if and only if the polynomial (6) has a root in the open right-half complex plane $\mathbb{C}_{+}$.

Proof. Let $u_{i}:=f_{i}\left(p_{i-1} / K_{i-1}\right)$. The model (5) can be viewed as a linear time-invariant system with the input $u_{i}$ and the output $p_{i}$, since it is linear in terms of $u_{i}, r_{i}$ and $p_{i}$. The transfer function from $u_{i}$ to $p_{i}$ is then obtained as

$$
g_{i}(s):=\frac{R_{i}^{2}}{\left(T_{a_{i}} s+1\right)\left(T_{b_{i}} s+1\right)} .
$$

Linearizing $u_{i}$ around $p_{i-1}=p_{i-1}^{*}$, we have

$$
u_{i}=f_{i}^{\prime}\left(\frac{p_{i-1}^{*}}{K_{i-1}}\right)\left(p-p_{i-1}^{*}\right)
$$

with

$$
\begin{aligned}
f_{i}^{\prime}\left(\frac{p_{i-1}^{*}}{K_{i-1}}\right) & =-\frac{1}{K_{i-1}} \frac{\nu_{i} \frac{p_{i-1}^{*}}{K_{i-1}}}{\left(1+\left(\frac{p_{i-1}^{*}}{K_{i-1}}\right)^{\nu_{i}}\right)^{2}} \\
& =\frac{1}{K_{i-1}} f_{i}^{\prime}\left(\frac{p_{i-1}^{*}}{K_{i-1}}\right) .
\end{aligned}
$$

As a result, the linearized system can be described by the block diagram shown in Fig. 2, and the characteristic equation of the closed loop system is obtained as

$$
1-\prod_{i=1}^{n} g_{i}(s) \frac{1}{K_{i}} f_{i}^{\prime}\left(\frac{p_{i-1}^{*}}{K_{i-1}}\right)=0,
$$

which leads to the equation (6).

The first term of the characteristic polynomial (6) depends only on the degradation rates of mRNAs and proteins. Thus, the choice of promoters and RBSs affects only the second term of the characteristic polynomial. In the second term, the constant $R_{i}^{2}$ is the ratio of production rates and degradation rates. In particular, $R_{i}^{2}$ can be interpreted as the gain of gene $\mathcal{G}_{i}$ from the definition of $g_{i}(s),(8)$.

Since the system is a simple cyclic feedback without branches as shown in Fig. 2, the second term

$$
G:=\prod_{i=1}^{n}\left(\frac{R_{i}^{2}}{K_{i}}\right) f^{\prime}\left(\frac{p_{i-1}^{*}}{K_{i-1}}\right)
$$

is an effective loop gain of the system. Thus, increasing the loop gain $G$ leads to instability of the equilibrium point. From a biological viewpoint, it is intuitive that $R_{i}^{2} / K_{i}$ is part of the loop gain as increasing $R_{i}^{2}$ implies sufficient production of repressors and decreasing $K_{i}$ allows for strong binding of the repressor to the promoter. However, too much production of repressors, or too large $R_{i}^{2}$, causes the saturation of the repressors and results in a non-oscillatory steady state. This tradeoff is captured by the derivative of the Michaelis-Menten kinetics $f^{\prime}(\cdot)$ around the operation point $p_{i-1}^{*}$, which depends on $R_{i}^{2} / K_{i}$ as shown below.

To calculate the equilibrium point, let $\dot{r}_{i}=\dot{p}_{i}=0(i=$ $1,2, \cdots, n)$. It follows from the model (5) that the equilibrium point satisfies

$$
p_{i}^{*}=R_{i}^{2} f_{i}\left(\frac{p_{i-1}^{*}}{K_{i-1}}\right) .
$$

This leads to the following equation that the equilibrium point should satisfy.

$$
\begin{aligned}
\frac{p_{i}^{*}}{K_{i}}= & \left(\frac{R_{i}^{2}}{K_{i}} f_{i}\right) \circ\left(\frac{R_{i-1}^{2}}{K_{i-1}} f_{i-1}\right) \circ \cdots \circ\left(\frac{R_{1}^{2}}{K_{1}} f_{1}\right) \\
& \circ\left(\frac{R_{N}^{2}}{K_{N}} f_{N}\right) \circ \cdots \circ \frac{R_{i+1}^{2}}{K_{i+1}} f_{i+1}\left(\frac{p_{i}^{*}}{K_{i}}\right),
\end{aligned}
$$

where $\circ$ stands for composition of functions.

Lemma 2. The loop gain $G$ depends only on the ratios $R_{i}^{2} / K_{i}(i=1,2, \cdots, n)$.

The proof is straightforward from the equation (14) and the characteristic polynomial (6). This lemma implies that one needs to consider only the ratios $R_{i}^{2} / K_{i}$ but not individual rates of transcription and translation. Therefore, the design space of promoters and RBSs can be mapped into a lower dimensional parameter space with $R_{i}^{2} / K_{i}(i=1,2, \cdots, n)$ as shown below.

\section{B. Effect of heterogeneous expression kinetics between genes}

The analysis of oscillatory parameter regime boils down to the analysis of the relation between

$$
v:=\prod_{i=1}^{n} \frac{R_{i}^{2}}{K_{i}},
$$

which appears in the definition of the loop gain (12), and the equilibrium point calculated by the equation (14). One of the important questions is how the heterogeneity of the parameters between genes affects the loop gain $G$. More specifically, consider an oscillator design problem under the constraint of $v=v_{0}$, where $v_{0}$ is a given constant. It is clear that the combinations of $R_{i}^{2} / K_{i}(i=1,2, \cdots, n)$, or promoter and RBS pairs, that satisfy the constraint is not unique. Then, the question is whether we should design a circuit with genetic parts that have similar ratios of $R_{i}^{2} / K_{i}$ or not, in order to produce oscillations.

We studied the effect of heterogeneity by solving the characteristic equation (6) with varying $R_{i}^{2} / K_{i}$ for $n=3$ gene oscillators. Figure 3 illustrates the largest real part of 


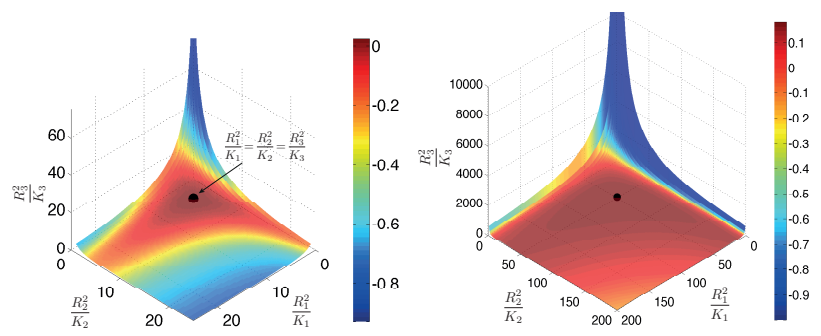

Fig. 3. The largest real part of the roots of the characteristic polynomial (6) with $\nu_{i}=2(i=1,2,3)$. (Left) $v_{0}=5.0$. (Right) $v_{0}=50.0$

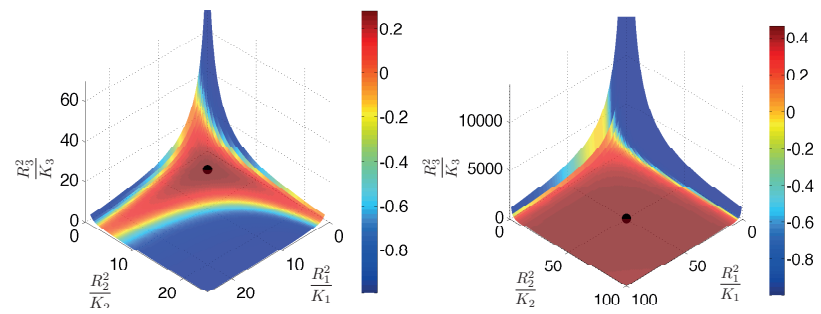

Fig. 4. The largest real part of the roots of the characteristic polynomial (6) with $\nu_{i}=3(i=1,2,3)$. (Left) $v_{0}=5.0$. (Right) $v_{0}=50.0$

the roots of the characteristic equation for Hill coefficient $\nu_{1}=\nu_{2}=\nu_{3}=2.0$. The left and the right figures correspond to the case of $v=5.0$ and $v=50.0$, respectively. It should be noted that the gene regulatory network has oscillations when the largest real part of the roots is positive (see Proposition 1 and Lemma 1).

Figure 3 shows that the system tends to be unstable as the gains of genes, $R_{i}^{2} / K_{i}(i=1,2,3)$, are close to each other, which is shown by a black point in the figure. The same conclusion holds for $\nu_{1}=\nu_{2}=\nu_{3}=3.0$ as illustrated in Fig. 4. These results suggest that oscillator circuits should be designed so that $R_{i}^{2} / K_{i}$ are similar to each other.

An interpretation of this conclusion is that repressors are saturated when the expression kinetics of genes are unbalanced, and consequently the oscillations are diminished due to the low sensitivity of the promoter activity. Thus, it is better to prioritize balancing $R_{i}^{2} / K_{i}(i=1,2, \cdots, n)$ rather than increasing a single value of $R_{i}^{2} / K_{i}$ to increase the product $v$.

\section{INTRINSINC NOISE OF OSCILLATORS}

We have shown in the previous section that it is important to balance the kinetics of expression between genes to produce oscillations. Motivated by this result, we further analyze the dynamics of cyclic gene regulatory networks with the following homogeneous assumption.

Assumption 2. We assume $a_{1}=a_{2}=\cdots=a_{n}(=: a), b_{1}=$ $b_{2}=\cdots=b_{n}(=: b), c_{1}=c_{2}=\cdots c_{n}(=: c), \beta_{1}=\beta_{2}=$ $\cdots=\beta_{n}(=: \beta), K_{1}=K_{2} \cdots=K_{n}(=: K)$ and $\nu_{1}=\nu_{2}=$ $\cdots=\nu_{n}(=: \nu)$.

\section{A. Existence of oscillations}

Hori et al. [15] studied the relation between the parameters and the equilibrium point with Assumption 2 and analytically

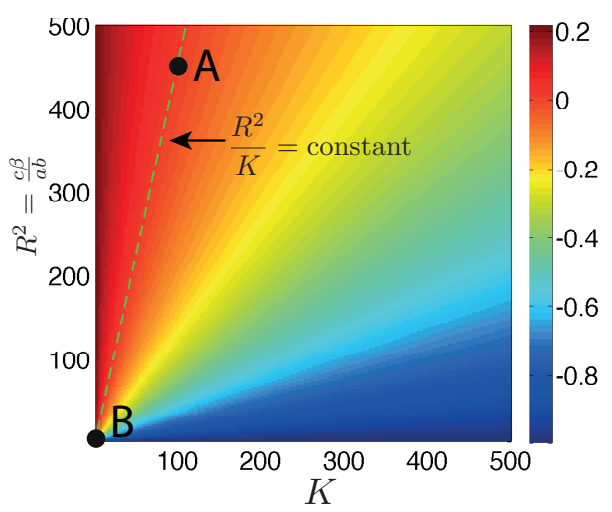

Fig. 5. The largest real parts of the characteristic polynomial (6).

derived a closed form condition for local instability as follows.

Proposition 2. [15] The characteristic polynomial (6) has at least one root in the open right-half complex plane $\mathbb{C}_{+}$if and only if

$$
\begin{aligned}
& \nu>h(n, Q), \\
& \frac{R^{2}}{K}>\left(\frac{h(n, Q)}{\nu-h(n, Q)}\right)^{\frac{1}{\nu}},\left(\frac{\nu}{\nu-h(n, Q)}\right),
\end{aligned}
$$

where

$$
\begin{aligned}
& Q:=\frac{\sqrt{a b}}{(a+b) / 2}, R^{2}:=\frac{c \beta}{a b} \\
& h(n, Q):=\frac{2\left(-\cos \left(\frac{\pi}{n}\right)+\sqrt{\cos ^{2}\left(\frac{\pi}{n}\right)+Q^{2} \sin ^{2}\left(\frac{\pi}{n}\right)}\right)}{Q^{2} \sin ^{2}\left(\frac{\pi}{n}\right)} .
\end{aligned}
$$

It should be noted that the right-hand side of the inequality (17) does not depend on $R^{2}$ and $K$. This leads to the conclusion that it is better to simply make $R^{2} / K$ as large as possible.

Example 1: Figure 5 illustrates the largest real part of the roots of the characteristic polynomial (6) for cyclic gene regulatory networks with $n=3$ genes and $Q=1.0$. Proposition 1 states that the cyclic gene regulatory network has oscillations when the largest real part is positive. As implied by the condition (17), the largest real part depends only on $R^{2} / K$, thus it is invariant as long as $R^{2} / K$ is constant, which is shown by the dotted line in Fig. 5.

Proposition 2 allows us to design oscillator circuits using the parameter set $A$ and $B$ in Fig. 5. The circuit design $A$ has large $R^{2}$, i.e., a relatively strong RBS and promoter, with a large Michaelis-Menten constant $K$, and the circuit design $B$ has small $R^{2}$, i.e., a relatively weak RBS and promoter, with a small $K$. Note that the real part of the right-most unstable poles are identical for the designs $A$ and $B$, meaning that the degree of local instability is the same. However, it is not clear from the stability analysis that how the choice of the transcription rate $c$, the translation rate $\beta$ and the MichaelisMenten constant $K$ affects the dynamics of the cyclic gene 


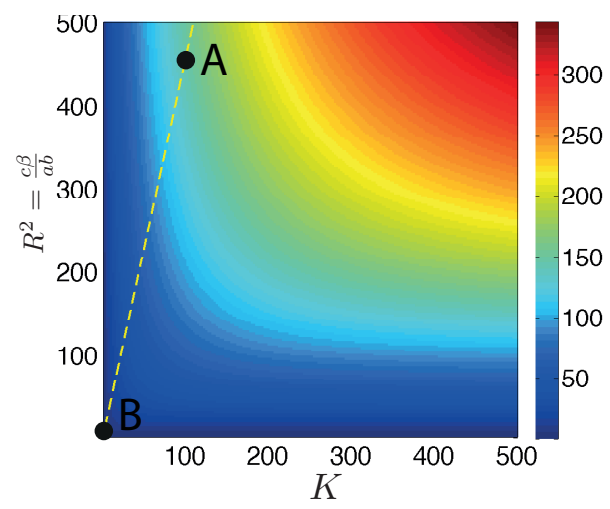

Fig. 6. Equilibrium protein concentration $p^{*}$.

regulatory networks when the resulting constant $R^{2} / K$ is the same between different designs.

In what follows, we address this problem from a viewpoint of intrinsic noise of biochemical reactions.

\section{B. Relation between protein copy numbers and Michaelis- Menten constant}

We here focus on the relation between the equilibrium point and the parameter $R^{2} / K$. The following lemma simplifies the calculation of the equilibrium point.

Lemma 3. Suppose Assumption 2 holds. Then, the unique equilibrium point satisfies $p_{1}^{*}=p_{2}^{*}=\cdots=p_{n}^{*}\left(=: p^{*}\right)$ and $r_{1}^{*}=r_{2}^{*}=\cdots=r_{n}^{*}\left(=: r^{*}\right)$. Moreover, it holds that

$$
\begin{aligned}
p^{*} & =R^{2} f\left(\frac{p^{*}}{K}\right), \\
r^{*} & =\frac{\beta}{a} f\left(\frac{p^{*}}{K}\right),
\end{aligned}
$$

where $f(\cdot):=f_{1}(\cdot)=f_{2}(\cdot)=\cdots=f_{n}(\cdot)$.

Proof. Since Assumption 2 holds, the right-hand side of the equation (13) becomes exactly the same for all $i=$ $1,2, \cdots, n$. This symmetric property implies $p_{1}^{*}=p_{2}^{*}=$ $\cdots=p_{n}^{*}\left(=: p^{*}\right)$. The rest of the proof is a direct calculation of the equilibrium point based on the definition.

Since $f(\cdot)$ is a decreasing function of $p^{*}$, the equilibrium point $p^{*}$ is given by the intersection of the left-hand side and the right-hand side of the equation (18). In particular, the right-hand side is an increasing function of $R^{2}$ and $K$. This means that the equilibrium concentration of $p^{*}$ is increasing in terms of $R^{2}$ and $K$. Since the orbit of a limit cycle revolves around an equilibrium point in nonlinear systems, oscillations are expected at higher concentrations, or higher copy number of proteins, as $R^{2}$ and $K$ increase. This suggests that one should choose the parameter set $A$ but not $B$ to reduce the intrinsic noise, which comes from the low-copy-number nature of the molecules in a cell.

Corollary 1. The equilibrium concentration of the proteins $p^{*}$ increases with $K$ and $R^{2}$.

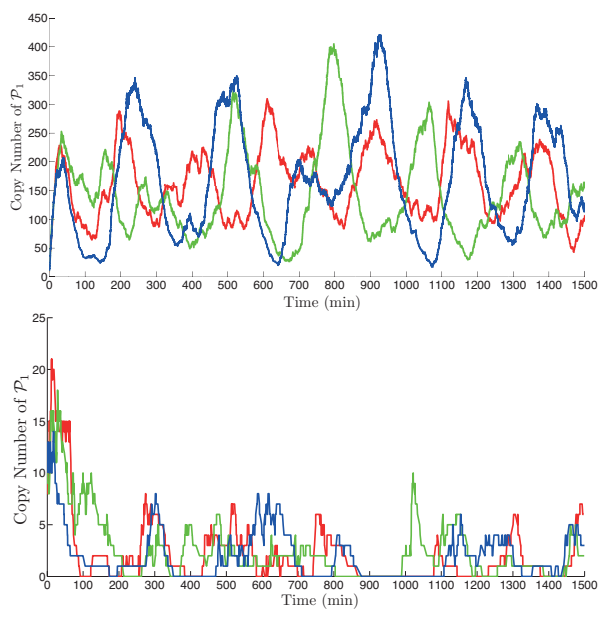

Fig. 7. Dynamics of the copy number of protein $\mathcal{P}_{1}$.

Example 2: Figure 6 illustrates the equilibrium protein concentration $p^{*}$ in terms of $K$ and $R^{2}$ using the same parameters as Example 1. The equilibrium point $p^{*}$ monotonically increases with $K$ and $R^{2}$ as discussed above. The dotted line showing a constant $R^{2} / K$ in Fig. 5 is also plotted in Fig. 6.

To compare the waveform of oscillations, the cyclic gene regulatory network was simulated for the parameter sets $A$ and $B$ using the stochastic simulation algorithm (SSA) [21]. The stoichiometry and propensity functions in Table I and the following parameters were used for the simulations: the number of genes $n=3$, the degradation rate of mRNA $a=$ $\ln (2) / 10$, i.e., half-life time is 10 minutes, the degradation rate of protein $a=\ln (2) / 20$, i.e., half-life time is 20 minutes, Hill coefficient $\nu=2$. At $t=0$, mRNA $\mathcal{M}_{1}$ and protein $\mathcal{P}_{1}$ were set as 10 copies each, and $\mathcal{M}_{2}, \mathcal{M}_{3}, \mathcal{P}_{2}$ and $\mathcal{P}_{3}$ were zero.

We simulated with the parameter sets $A$ and $B$ in Figs. 5 and 6. Specifically, we used transcription rate $\beta=1.0$, translation rate $c=1.1$ and Michaelis-Menten constant $K=$ 100 for the set $A$, and $\beta=0.10, c=0.11$ and $K=1$ for the set $B$.

The simulation results are shown in Figs. 7 (top) and (bottom) for the parameter set $A$ and $B$, respectively. Each figure shows three representative time courses of the copy number of $\mathcal{P}_{1}$. Although $R^{2} / K$ is the same for both $A$ and $B$, the copy number of the protein molecule is significantly large when the values of $R^{2}$ and $K$ are large, which is consistent with the equilibrium point of the deterministic model in Fig. 6. In particular, it is hard to see oscillatory dynamics for the parameter set $B$, or Fig. 7 (bottom), due to the fluctuations by intrinsic noise.

\section{Tradeoff between $m R N A$ and protein copy numbers}

In the previous subsection, we have shown that larger $R^{2}$ and the Michaelis-Menten constant $K$ increases the copy number of proteins, which results in the reduction of the intrinsic noise of oscillations. Suppose we use the parameter set $A$ of Fig. 5, which is $K=100$ and $R^{2}=457.9$. We here 

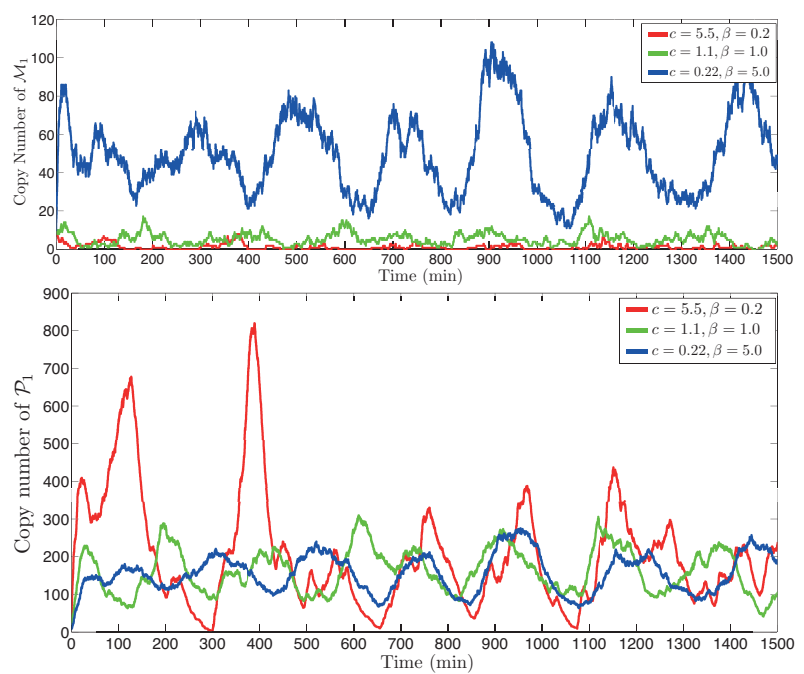

Fig. 8. (Top) Dynamics of the copy number of mRNA $\mathcal{M}_{1}$ with the different rates of transcription, $\beta$, and translation $c$. (Bottom) Dynamics of the copy number of protein $\mathcal{P}_{1}$ with the different rates of transcription, $\beta$, and translation $c$.

consider another design parameter, the rate of transcrpition $\beta$ and translation $c$. More specifically, given $R^{2}=c \beta / a b$, we analyze how the strength of promoter, $\beta$, and RBS, $c$ affects the oscillations. Note that increasing $\beta$ decreases $c$, and vice versa, for a given $R^{2}$.

We first show that the equilibrium point of mRNA concentrations is invariant as long as the value of $R^{2} / K$ is the same.

Corollary. 2 The equilibrium concentration of $m R N A r^{*}$ is invariant in terms of $R^{2} / K$.

Proof. It follows from the equation (18) that $p^{*} / K$ is invariant with $R^{2} / K$. The equation (19) then implies that $r^{*}$ is invariant with $R^{2} / K$.

This corollary means that the equilibrium point is the same for all combinations of $R^{2}$ and $K$ on the dotted straight of Fig. 6, which is not the case for proteins as discussed in the previous subsection (see Fig. 6). In particular, the equation (19) implies that the ratio of transcription rate and mRNA degradation rate $\beta / a$ essentially determines the equilibrium concentration of mRNA. Thus, we can independently tune $r^{*}$ and $p^{*}$ by first determining the ratio of $R^{2}$ and the MichaelisMenten constant $K$ for $p^{*}$ then transcription rate $\beta$.

Example 3. To elucidate the idea, we simulated the dynamics of mRNA and protein copy numbers using the parameter set $A$. Note that the deterministic model is locally unstable from Fig. 5, and the equilibrium protein concentration is determined from Fig. 6. To vary mRNA concentrations, we varied transcription and translation rates $\beta$ and $c$ so that $c \beta$ is constant. The other parameters and initial values were set the same as Example 2.

Figure 8 (top) and (bottom) show the dynamics of mRNA and protein copy numbers for three different combinations of $c$ and $\beta$. As the transcription rate $\beta$ becomes larger and the translation rate $c$ becomes smaller, the time course of the mRNA copy number shows clear oscillatory dynamics, while the protein copy number oscillates around the same equilibrium point. On the other hand, the amplitude of protein oscillations decreases with the decrease of translation rate $c$.

There seems to be a tradeoff relation between the amplitude of protein oscillations and the regularity of the oscillation period when $c \beta$ is constant. Specifically, increasing transcription rate $\beta$ results in smaller amplitude for protein oscillation as shown in Fig. 8. On the other hand, the period of oscillations seems more regular with the high transcription rate 5.0 and the low translation rate 0.22 compared to other parameter sets in Fig. 8. However, rigorous study of such tradeoff relations is left for future work.

\section{CONCLUSION}

In this paper, we have explored engineering principles of biochemical oscillators with negative cyclic feedback. We have first studied local instability of the unique equilibrium point using a combined analytic and numerical approach. Then, the latter half of the paper has been devoted to the analysis of intrinsic noise of the oscillator circuit using the lower dimensional parameter space derived from the analytic conditions. The analysis result can be summarized as follows.

- The parameters of gene expression $c, \beta$ and $K$ should be similar to each other.

- The existence of oscillations is determined by the ratio $R^{2} / K$ (Fig. 5).

- When $R^{2} / K$ is given, the larger Michaelis-Menten constant $K$ and $R^{2}$ are, the larger $p^{*}$ is (Fig. 6). Thus, the effect of intrinsic noise is less with larger $K$ and $R^{2}$.

- When $R^{2} / K$ is given, the steady state mRNA level $r^{*}$ is linear in terms of transcription rate $\beta$, or the strength of promoter. In particular, there seems to be a tradeoff relation between the amplitude of protein oscillations and the regularity of the oscillation period when $c \beta$ is constant.

\section{REFERENCES}

[1] M. B. Elowitz and S. Leibler, "A synthetic oscillatory network of transcriptional regulators," Nature, vol. 403, no. 6767, pp. 335-338, 2000.

[2] E. Franco et al., "Timing molecular motion and production with a synthetic transcriptional clock," Proceedings of the National Academy of Sciences, vol. 108, no. 40, pp. E784-E793, 2011.

[3] J. Stricker et al., "A fast, robust and tunable synthetic gene oscillator," Nature, vol. 456, no. 7221, pp. 516-519, 2008.

[4] M. R. Atkinson et al., "Development of genetic circuitry exhibiting toggle switch or oscillatory behavior in Escherichia coli," Cell, vol. 113, no. 7221, pp. 597-607, 2003.

[5] B. C. Goodwin, "Oscillatory behavior in enzymatic control process," Advances in Enzyme Regulation, vol. 3, pp. 318-356, 1965.

[6] F. Jacob and J. Monod, "Genetic regulatory mechanisms in the synthesis of proteins," Journal of Molecular Biology, vol. 3, no. 6, pp. 318-356, 1961.

[7] J. J. Tyson, "On the existence of oscillatory solutions in negative feedback cellular control processes," Journal of Mathematical Biology, vol. 1, no. 4, pp. 311-315, 1975. 
[8] P. Rapp, "Analysis of biochemical phase shift oscillators by a harmonic balancing technique," Journal of Mathematical Biology, vol. 25, no. 3, pp. 203-224, 1976.

[9] S. Hastings, J. Tyson, and D. Webster, "Existence of periodic solutions for negative feedback cellular control systems," Journal of Differential Equations, vol. 25, pp. 39-64, 1977.

[10] C. D. Thron, "The secant condition for instability in biochemical feedback control - partI, II," Bulletin of Mathematical Biology, vol. 53, no. 3, pp. 383-401, 1991.

[11] H. T. Banks and J. Mahaffy, "Stability of cyclic gene models for systems involving repression," Journal of Theoretical Biology, vol. 74, no. 2, pp. 323-334, 1978.

[12] D. Angeli and E. D. Sontag, "Oscillations in I/O monotone systems under negative feedback," IEEE Transactions on Automatic Control, vol. 53, no. special issue, pp. 166-176, 2008.

[13] M. Arcak and E. D. Sontag, "Diagonal stability of a class of cyclic systems and its connection with the secant criterion," Automatica, vol. 42, no. 9, pp. 1531-1537, 2006.

[14] — "A passivity-based stability criterion for a class of biochemical reaction networks," Mathematical Biosciences and Engineering, vol. 5, no. 1, pp. 1-19, 2008.

[15] Y. Hori, T.-H. Kim, and S. Hara, "Existence criteria of periodic oscillations in cyclic gene regulatory networks," Automatica, vol. 47, no. 5, pp. 1203-1209, 2011.

[16] Y. Hori, M. Takada, and S. Hara, "Biochemical oscillations in delayed negative cyclic feedback: existence and profiles," Automatica, vol. 49, no. 9, pp. 2581-2590, 2013.

[17] B. C. Stanton et al., "Genomic mining of prokaryotic repressors for orthogonal logic gates," Nature Chemical Biology, vol. 10, no. 2, pp. 99-105, 2014.

[18] V. K. Mutalik et al., "Precise and reliable gene expression via standard transcription and translation initiation elements," Nature Methods, vol. 10, no. 4, pp. 354-360, 2013.

[19] H. L. Smith, "Oscillations and multiple steady states in a cyclic gene model with repression," Journal of Mathematical Biology, vol. 5, no. 2, pp. 169-190, 1987.

[20] J. Mallet-Paret and H. L. Smith, "The Poincaré-Bendixson theorem for monotone cyclic feedback systems," Journal of Dynamics and Differential Equations, vol. 2, no. 4, pp. 367-421, 1990.

[21] D. T. Gillespie, "Exact stochastic simulation of coupled chemical reactions," Journal of Physical Chemistry, vol. 81, no. 25, pp. 23402361, 1977. 